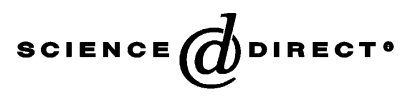

Behavioural Processes 62 (2003) 125-144

\section{BeHAVIOURAL Processes}

www.elsevier.com/locate/behavproc

\title{
Successive two-item same-different discrimination and concept learning by pigeons
}

\author{
Robert G. Cook*, Debbie M. Kelly, Jeffrey S. Katz \\ Department of Psychology, 530 Bacon Hall, Tufts University, Medford, MA 02155, USA
}

Received 23 August 2002; accepted 18 December 2002

\begin{abstract}
Four pigeons were trained in a successive same/different procedure involving the alternation of two stimuli per trial. Using a go/no-go procedure, two different or two identical color photographs were alternated, with a brief, dark, inter-stimulus interval, on a computer screen for $20 \mathrm{~s}$. Pigeons learned to discriminate between same (S+) and different (D-) sequences with moderate to large contrasts between successive pictures. Analyses of pecking behavior within single trials revealed this discrimination emerged at the earliest possible point in the sequence (i.e. by the presentation of the second item). Pigeons transferred to novel color and gray-scale pictures, and showed savings in tests with novel video stimuli. These results suggest that same/different discrimination and concept formation can be acquired with successively presented pairs of stimuli by pigeons. When combined with results using simultaneous same/different presentations, these findings further support a qualitative similarity among birds and primates in their capacity to judge certain types of stimulus relations.
\end{abstract}

(C) 2003 Elsevier Science B.V. All rights reserved.

Keywords: Pigeon; Concept learning; Discrimination training

The importance of understanding animal conceptual behavior continues to be a key issue in the comparative study of behavior, thinking, and intelligence (see Cook, 2001 for many examples). The ability to abstract and recognize the regular structure and patterns of objects and events is critical to any animal predicting what might happen next. In recent years, our research has focused on how pigeons discriminate and potentially conceptualize the regularities associated with same and different (S/D) relations among sets of visual elements. This ability is valuable because the generalized recognition of difference and identity is a fundamental psychological discrimination that is

\footnotetext{
* Corresponding author. Tel.: +1-617-627-2456; fax: +1-617-627-4132.

E-mail address: rcook1@tufts.edu (R.G. Cook).

$U R L:$ http://www.pigeon.psy.tufts.edu
}

central to many advanced intellectual and artistic activities such as language, mathematics, sequential behavior, analogical reasoning, social relations, music, and painting.

In a S/D task, the subject has to respond "same" when two or more stimuli are identical and "different" if one or more of the stimuli are different from the others. After learning this discrimination, the degree to which this behavior transfers to novel situations having same and different relations is taken as evidence of concept formation. Using this choice task, it has been found that pigeons, parrots, rhesus monkeys, baboons, and chimpanzees are capable of learning and applying a S/D concept across a wide variety of simultaneously presented visual elements (Bovet and Vauclair, 2001; Cook, 2002a,b; Cook et al., 1995, 1997, 1999; Cook and Wixted, 1997; Katz et al., 2002; Pepperberg, 1987; Thompson et al., 1997). 
In our studies we have found that pigeons can learn a S/D classification with up to five different multidimensional classes, ranging from smaller, densely packed textural elements to more sparse arrays of geometric shapes, objects (e.g. birds, flowers), and photographs (Cook, 2002b; Cook et al., 1997, 1999; on-line examples of these stimulus classes can been found at http://www.pigeon.psy.tufts.edu). Further, the pace of S/D learning with these different stimulus classes proceeds at the same rate, suggesting that the same common discrimination framework or decision criterion is applied across all of these distinct types (Cook, 2002b; Cook et al., 1997). In addition to learning a single, broadly applied rule, we have consistently found the pigeons transfer this solution to novel exemplars from both within (Cook et al., 1995, 1997) and outside the range of stimuli experienced during training (Cook et al., 1999). This latter kind of transfer is important as the greater the range of novel conditions over which a discrimination can be applied, the more abstract a conceptual representation is required. As a whole, these results seem most consistent with the hypothesis that pigeons can detect, recognize, and abstract simultaneously presented S/D visual relations.

Are alternative accounts possible? One possibility is that perceptual processing of the same and different arrays directly mediates performance rather than a conceptual representation of these relations (e.g. Mackintosh, 2000). For example, in texture-based S/D displays, discrimination could be based on detection of the presence or absence of a square border contrast between the target and distractor region (Cook et al., 1995). If true, then pigeons could successfully discriminate novel displays based on the detectable presence or absence of this simple perceptual feature and not on an abstract representation of its component relations.

We have discounted this type of explanation in part because novel test items consistently show lower discrimination than with baseline training items. If only perceptual information about spatial discontinuities mattered, then why should element familiarity be important? Second and more compelling, pigeons exhibit good discrimination transfer even when tested with S/D relations constructed from distinctively different stimulus classes (Cook, 2002b; Cook

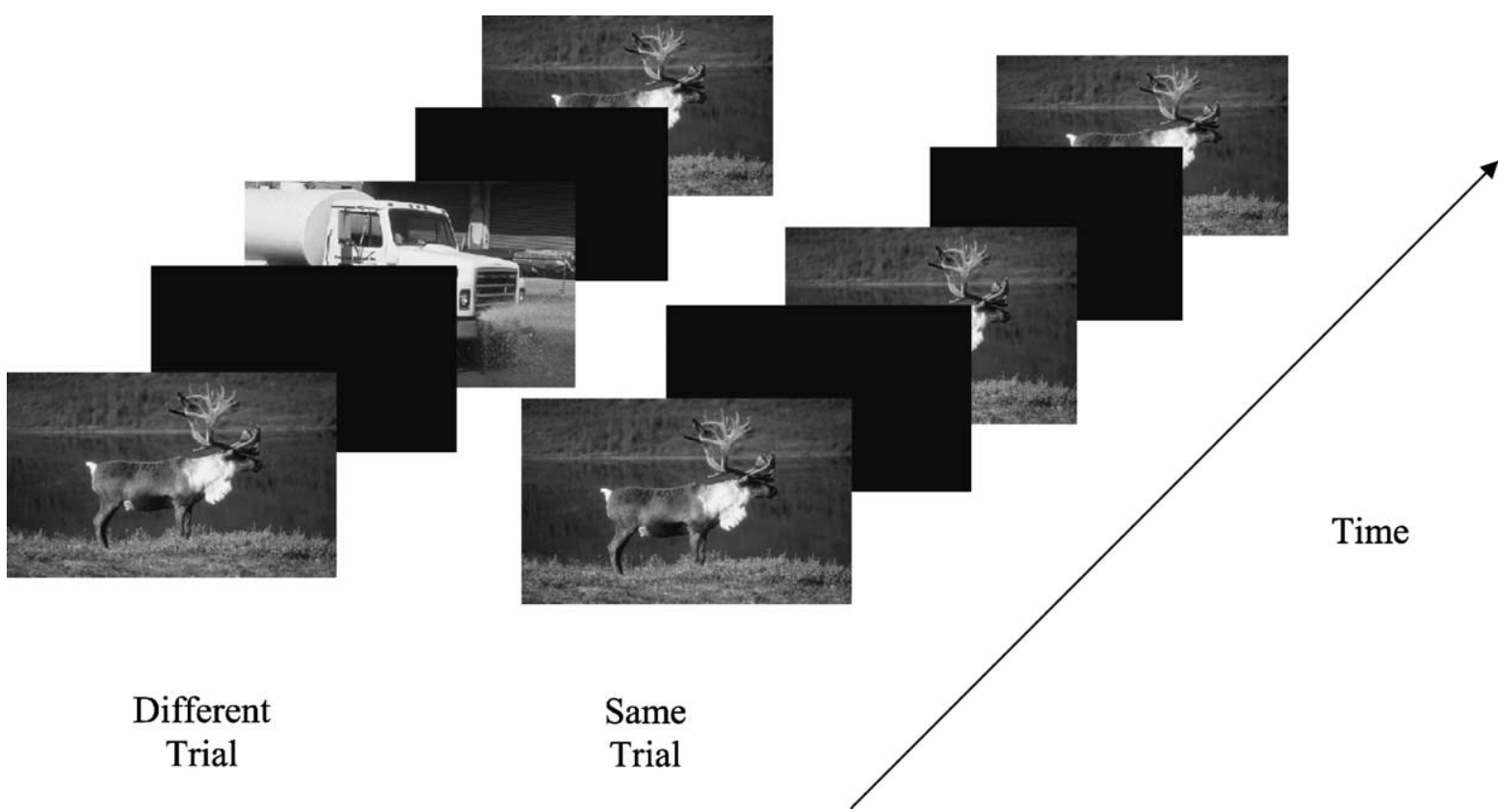

Fig. 1. A schematic representation of the same and different sequences tested in these experiments. Each item appeared for a fixed period of time and was separated by a dark inter-stimulus interval. Each sequence was presented for $20 \mathrm{~s}$ and pecks to same sequences were reinforced on a VI 10-s schedule, while pecks to different sequences were punished by a brief timeout. 
et al., 1997, 1999) where any simple feature-based alternative seems far more implausible. Nevertheless, because we have previously only employed simultaneously-presented, multiple-element displays that by their very nature must have a spatial contrast on the different trials, we thought it important to test a S/D procedure that eliminates this feature.

In the experiments reported in this article, we tested pigeons using a go/no-go discrimination, in which alternating sequences of either same (AAAA ... or BBBB ... ) or different (ABAB ... or BABA ...) photographic stimuli were presented within a trial (see Fig. 1). At any one time only a single item was visible, thereby eliminating any perceptual features related to element simultaneity as a basis for learning or transfer. We were also quite interested in seeing if comparisons involving only two items were capable of generating S/D behavior, as our previous experiments had always used multiple elements in constructing the displays.

This latter issue is particularly interesting because previous research with pigeons using successively presented displays in a S/D task used many identical or contrasting stimuli within a trial to generate discriminative behavior (Young et al., 1997, 1999). In Young et al.'s research, pigeons were tested with successive presentations of either 16 same or 16 completely different small icons (one peck per item), after which the animals were required to make a "same" or "different" choice response. After learning this multiple-item discrimination, Young et al. (1999) found that when tested with only two alternating items, the pigeons failed to discriminate the difference between the items, and instead consistently responded "same" to such sequences. One possible reason for this is that their pigeons were initially trained with long sequences in which every item was either identical or different from one another. Young and Wasserman have consistently found that this type of training results in the pigeons learning to be controlled by item variability or the "entropy" within the displays. By conducting our S/D training from the beginning with alternating two-item sequences, we hoped to eliminate control by this continuous variability dimension and provide instead a basis for the pigeons to learn a more categorical S/D judgment about the relations between successively presented items.

\section{Experiment 1}

The objective of Experiment 1 was to see if pigeons could learn a successive S/D discrimination involving only two alternating stimulus items. We tested four naïve pigeons with either same or different sequences of photographs in a go/no-go task. At the start of the experiment, one of our ancillary objectives was also to develop potential procedures for evaluating "change blindness" (e.g. Rensink et al., 1997) in pigeons. Change blindness is the human failure to detect visual change in a scene without attention to the specific locus of change. This phenomenon has been a springboard for new insights into how humans represent the visual details of a scene (reviewed by Simons, 2000) and we hoped to address similar issues in pigeons. Because humans' find it difficult to detect change, we chose to use a method where the same sequences were always the reinforced $\mathrm{S}+$ condition and the different sequences were the non-reinforced condition (D-, i.e. a D+/S- condition was not included). Furthermore, we used semi-artificial stimuli consisting of synthetic combinations of natural backgrounds and small object figures, that created both large and small changes between the successive items of a sequence. Using a discrete-trials procedure, each same or different sequence of alternating items was presented for $20 \mathrm{~s}$. During this presentation time, individual items were presented for either a 1 or $3 \mathrm{~s}$ viewing duration and separated by either a 100 or $500 \mathrm{~ms}$ dark inter-stimulus interval (ISI). The basic question was whether the pigeons could learn this task.

\subsection{Method}

\subsubsection{Animals}

Four naïve male White Carneaux pigeons (Columba livia) were tested. They were maintained at $85 \%$ of their free-feeding weights in a colony room with a 12:12 light/dark cycle and had free access to water and grit in their home cages.

\subsubsection{Apparatus}

Testing was done in a flat-black Plexiglas chamber $(38 \mathrm{~cm}$ wide $\times 36 \mathrm{~cm}$ deep $\times 38 \mathrm{~cm}$ high). The stimuli were presented on a color computer monitor (NEC MultiSync C500; McDonough, GA) visible through a $26 \mathrm{~cm} \times 18 \mathrm{~cm}$ viewing window in the middle of the 
front panel of the chamber. The viewing window's bottom edge was $20 \mathrm{~cm}$ above the chamber floor. The monitor was protected by a thin piece of glass mounted in this window. Pecks to the monitor were detected by an infrared LED touch screen (resolution of $80 \times 48$ locations; EMS Systems, Champaign, IL) mounted behind a $40 \mathrm{~mm}$ wide Plexiglas ledge that went around the inside edge of the viewing window. A house light was located in the ceiling of the chamber and was illuminated at all times, except during timeouts. Mixed grain was delivered by a food hopper that was centrally located in the front panel with its access hole flush to the floor of the chamber. All experimental events were controlled and recorded by a computer. A graphics card operated the monitor at an $800 \times 600$ pixel, 16-bit color resolution. Computer-controlled relays (Metrabyte, Taunton, MA) operated the hopper and house light.

\subsubsection{Procedure}

Pigeons were first autoshaped to peck at a white warning signal $(30 \mathrm{~mm}$ in diameter). Once consistent responding was established, sequences of stimuli were introduced. Each of these trials started with a single peck to the warning signal, followed by either an alternating sequence of either identical (AAAA ... same trials) or different (ABAB ... different trials) photographic stimuli for $20 \mathrm{~s}$. During each $20 \mathrm{~s}$ presentation, each stimulus item was presented for either 1000 or $3000 \mathrm{~ms}$ viewing duration, followed by either a 100 or $500 \mathrm{~ms}$ dark ISI, and then the next matching or different picture. Only one viewing time and ISI was used within a trial. During the first six to eight sessions both types of sequences were reinforced on an increasingly lean variable-interval (VI) schedule until stable peck rates were established for three sessions using a VI 10 -s schedule. Food hopper presentations were $2.5 \mathrm{~s}$ in duration.

\subsubsection{Discrimination training}

The stimuli tested during discrimination training consisted of combinations of two elements-a small object figure centrally located on a naturalistic background. The figure elements consisted of pictures $(160 \times 160$ pixel) of six common objects (soccer ball, bell, key, blue cup, teddy bear, and black phone). The background elements consisted of pictures $(480 \times 300$ pixel) of six landscapes (sandy beach, rocky outcrop- ping, open barren field, deciduous woods, coniferous forest, stormy desert). A seventh background consisted of a simple uniform light blue rectangle.

Eight sequence conditions using these stimuli were tested. There were five different conditions consisting of alternating sequences of two contrasting stimuli. In the redundant condition both the figure and background elements change between successive stimuli; in the background condition the background element, but not the figure element, changed between stimuli; in the figure condition, the figure, but not the background element, changed; in the background-only condition the background changed between stimuli and no figure element was present in either; and in the figure-only condition the figure element changed between stimuli and the background was light blue stimulus field in both. These conditions were compared against three same conditions that consisted of alternating sequences of the identical picture. In the both same condition the figure and ground elements were the same across successive stimuli; in the background-only same condition, the background was the same across stimuli and no figure was present; and in the figure-only same condition the figure element was the same and the background was the uniform light blue field. The both same condition served as the comparison for the redundant, background, and figure different conditions. The background-only same condition served as the comparison for the background-only different condition, and the figure-only same condition as the comparison for the figure-only different condition. Within the definition of each condition, the component elements were randomly selected on each trial. Each session consisted of 120 trials. The 60 different trials consisted of the five different conditions tested 12 times each. Pecks to these sequences were never reinforced (D-). After the 12th session, a graded timeout, dependent on the number of pecks emitted within a trial (1.5 s per peck), was added as a consequence to these trials. To equate with the various different conditions, the 60 same trials consisted of testing the both same condition 36 times, and the remaining two conditions 12 times each, and pecks to these sequences were reinforced on a VI 10-s schedule. After the 37th session, $20 \%$ of the same trials were conducted as probe trials in which no reinforcement was delivered, allowing for the measurement of the $S+$ peck rates without the 
problems created by having reinforcements delivered within a trial. Twenty-four sessions were conducted with this modified procedure.

\subsection{Results}

All four pigeons easily learned the S/D task. Fig. 2 shows the acquisition over the first 36 sessions as measured by discrimination ratio (DR; $\mathrm{S}+$ pecks $/(\mathrm{S}+$ pecks $+\mathrm{D}-$ pecks $))$. The peck rates for the $\mathrm{S}+$ same trials were derived from those pecks emitted prior to the first delivery of reinforcement, while D- peck rates were based on the entire 20-s interval. Discrimination improved with the three S/D conditions that involved background changes, but showed no improvement in the conditions where the smaller object figure exclusively provided the critical information. A two-way repeated measures ANOVA (condition $\times$ four-session blocks) confirmed this interaction, $F(32,96)=4.6$, between conditions and sessions (all significance levels were judged against an alpha level of 0.05 ).

We next examined the effects of viewing duration and ISI by looking at the 24 sessions following the introduction of the $\mathrm{S}+$ probe trials. The pigeons continued to gradually improve during these sessions, but there was no change in the relations among the five conditions. Over these sessions, mean DRs in the redundant (71.9), background (72.2), and background-only (73.8) conditions were significantly above chance responding (50, single mean $t \mathrm{~s}(3)>$ 3.18 ), but figure (50.4) and figure-only (50.7) performance was not. Because of these small differences, we recombined these two clusters of conditions into just "figure" versus "background" in subsequent analyses. To judge the effects of item viewing duration and ISI we examined discriminative responding near the end of each trial, as this best measured the cumulative effect of the repeated cycles of these two temporal factors. Using peck rates over the last $5 \mathrm{~s}$ of each presentation, we found no significant effect of viewing duration on DR $(1000 \mathrm{~ms}=74.2,3000 \mathrm{~ms}=71.8$; $F(1,3)=3.6)$ for the "background" conditions, but a significant main effect of ISI, $F(1,3)=13.4$, with the short $100 \mathrm{~ms}$ (76.8) interval supporting a higher mean DR than the $500 \mathrm{~ms}$ interval (69.1). Not surprisingly, analyses of the figure conditions revealed no effect of either interval. We next looked in more

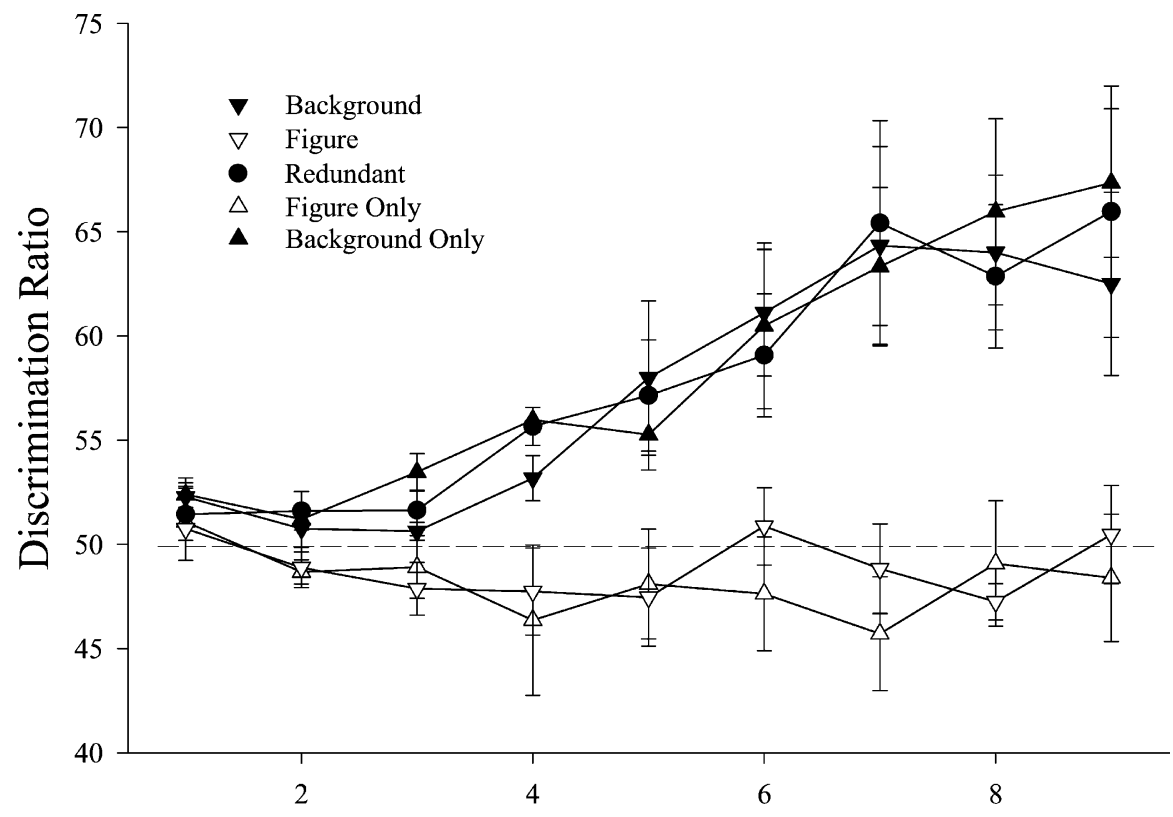

Four Session Blocks

Fig. 2. Mean discrimination ratio over the 36 sessions of training in four-session blocks for the five same/different conditions in Experiment 1. Error bars represent the standard error of the mean. The dashed line represents chance discrimination. 
detail at these effects using just the "background" conditions.

One important advantage of this successive go/nogo discrimination procedure is that it permits the on-line tracking of the discrimination within a trial, and thus revealing the microstructure of when and how these S/D sequences were discriminated by the pigeons. For the next analysis, we counted the number of pecks made during each individual item presentation within a trial. Because they may not have been under stimulus control, we excluded pecks made during ISIs. The pigeons tended not to peck the darkened screen anyway, and fewer than $10 \%$ of all pecks were made during the ISIs. Fig. 3 shows the results of this analysis. The top and middle panels show the mean number of pecks during each successive interval of the same and different sequences for each ISI and the $1000 \mathrm{~ms}$ (top panel) and $3000 \mathrm{~ms}$ (middle panel) viewing duration intervals. The bottom panel shows these same data replotted in terms of DR for each interval. There are several noteworthy features. The first and most important is that only a single stimulus transition was needed for the pigeons to begin differentially responding to the same and different sequences. That is, by the second stimulus presentation (the first stimulus item always being ambiguous as to whether it was a same or different sequence), the pigeons showed significant differences in peck rate to the second item with both the $1000, F(1,3)=22.58$, and $3000, F(1,3)=11.3$ viewing durations. Second, S/D information accumulated for at least $7000-8000 \mathrm{~ms}$ after the onset of the trial as DR improves over this time and then gradually flattens out. This result can be seen in the bottom panel for the 1000-100 and 1000-500 viewing duration-ISI conditions. In the 3000-100 and 3000-500 viewing duration-ISI conditions, DR seemed to asymptote around $13000 \mathrm{~ms}$. Measured in terms of stimulus transitions, it appears that S/D information accumulates up to three to six stimulus transitions depending on the stimulus presentation rate, with the 1000-ms conditions improving for more transitions than the 3000-ms conditions, but reaching its asymptote in a shorter period of time. One final note: because the pigeons could not differentially respond to the ambiguous first image, the analyses of later experiments were modified to exclude these pecks.

\subsection{Discussion}

The above experiment revealed that pigeons can learn a S/D task, at least when large areas of the stimuli change between stimuli, both when only two items are used to form the discrimination and these items are presented successively over time. They indicate that when sufficiently different S/D information is spread out across time, thereby eliminating spatial discontinuities and other perceptual features created by item simultaneity, pigeons can detect, compare, and respond to successively presented stimulus identity and difference. That some form of memory is involved in the comparison of these successive items is indicated by the fact that the longer ISI significantly hurt performance. Examination of the origins of the discrimination within a trial further established that only a single S/D transition was required to initiate differential behavior to same and different sequences. These analyses also revealed that such stimulus transitions further improved performance, independent of viewing and ISI timing, indicating that discriminative information accumulates within a trial. Viewing duration did not make a large contribution to the terminal level of the discrimination, but did affect how quickly the pigeons reached this level. The $1000 \mathrm{~ms}$ condition, with its faster presentation rate and increased number of transitions, reached its terminal level at around 7-10s, while the slower $3000 \mathrm{~ms}$ duration took about $12-13 \mathrm{~s}$ to reach the same level of discrimination. Further presentations made only very small increments to discrimination performance.

This description applies, however, only to those sequences in which large changes occurred between the items of the different trials. The pigeons consistently showed no capacity to discriminate the smaller, centrally located, object figures in these displays. Several possibilities for this difficulty can be considered. One is they failed to detect the figural changes either because of their smaller size in comparison to the background or their central location. Similar problems in detecting change or difference can occur in humans when focused attention is not specifically brought to the area of the display that is continuously changing (e.g. Rensink et al., 1997). A pure size account of this "change blindness" in pigeons seems unlikely because of the numerous studies that have found that pigeons can discriminate comparably sized stimuli, 

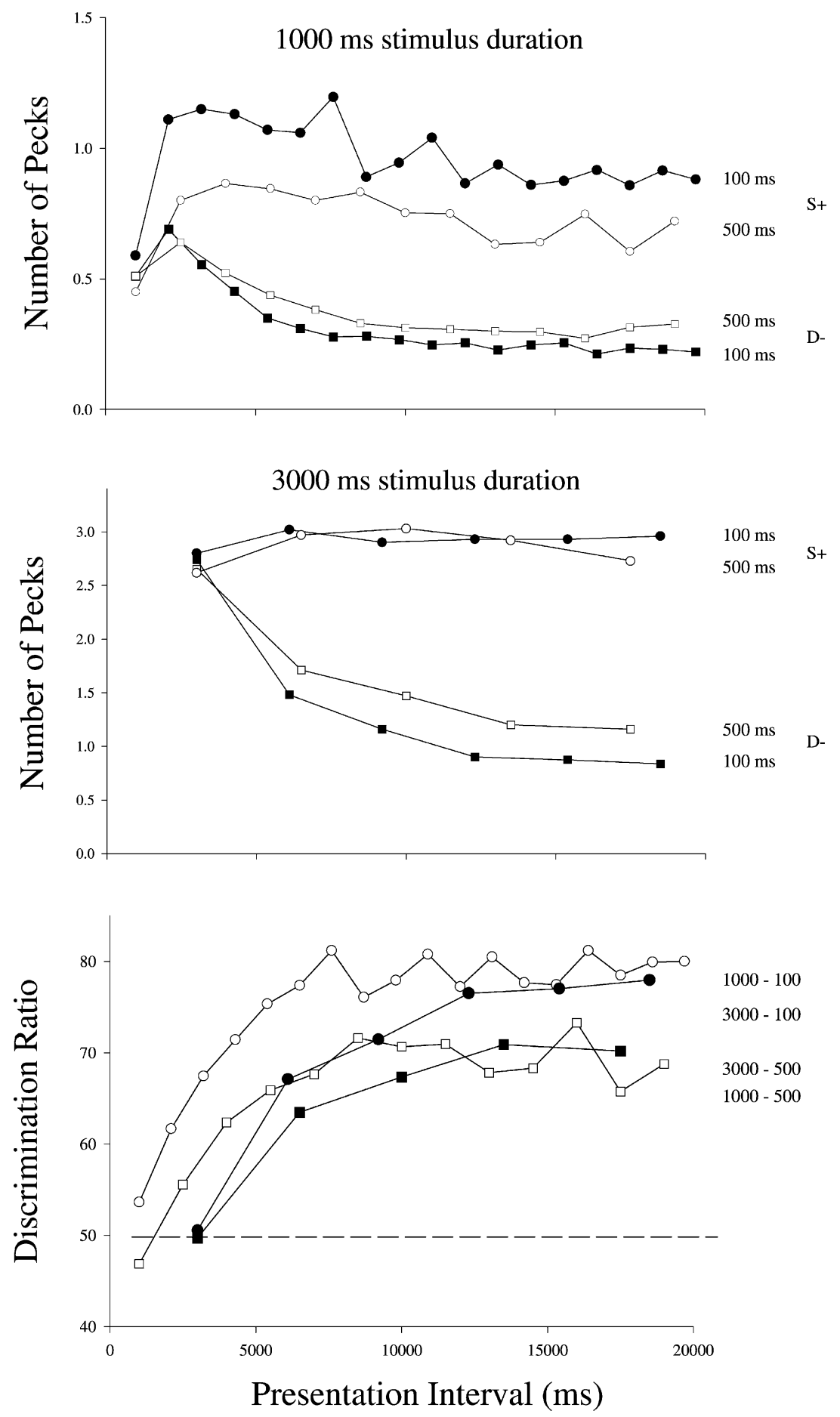

Fig. 3. Mean peck counts across successive intervals for same and different sequences of the combined background conditions during the second phase of Experiment 1. The top panel shows performance for each sequence type and ISI in the $1000 \mathrm{~ms}$ viewing condition. The middle panel shows performance for each sequence type and ISI in the $3000 \mathrm{~ms}$ viewing condition. Note the scale change between these two panels. The bottom panel shows performance as discrimination ratio for these conditions. In the labels the first number denotes stimulus duration and the second denotes the ISI. The dashed line represents chance discrimination. 
at least when pecked at directly by the birds (Carter and Werner, 1978; Cook et al., 1997; Wright, 1997; Young et al., 1997, 1999). The central location of the objects may have been more problematic. We found in analyzing where the pigeons pecked at the displays that they showed a strong tendency to peck at the outer portions of the left, right, and bottom edges of the displays (the bottom portion likely being attractive because of its proximity to the food hopper). If pigeons primarily process only what they peck at, then it is possible they never detected the contrasting objects in the center of the display, and thus preventing their discrimination (Cook et al., 1995; see Katz et al., 2002 for a similar argument with monkeys). A second possibility is that the pigeons did detect the figural changes between the stimuli, but that the proportion of change relative to their common background was not sufficient for the pigeons to consider it a "different" enough stimulus and respond accordingly.

\section{Experiment 2}

Experiment 2 looked further into the ISI effects observed in the first experiment. The ISI between the stimuli was systematically varied between 0 and $2 \mathrm{~s}$. If some form of memory for the sequence of items were involved, one should see a gradual and systematic effect of ISI, with performance decreasing with longer "retention intervals" between items.

\subsection{Method}

\subsubsection{Animals and apparatus}

The same pigeons and apparatus as in Experiment 1 were used.

\subsubsection{Procedure}

The organization of the same and different trials and the stimuli was the same as in Experiment 1, except that light blue background figure condition was omitted. Otherwise only the timing of the items in the same and different sequences was changed. First, the viewing duration per item was changed to a fixed $2000 \mathrm{~ms}$ for all trials. Second, the ISI between stimuli was systemically manipulated among trials (but remained constant within a trial). Each session consisted of 96 trials (48 same/48 different). During the 48 same trials, eight ISIs $(0,100,250,500,750$, $1000,1500,2000 \mathrm{~ms}$ ) were each tested six times. Two of these six trials for each ISI were probe trials in which no reinforcement was permitted. During the 48 different trials, the figure, redundant and background conditions were each tested 16 times, with each of the eight ISIs tested six times (twice per condition). Twenty-five sessions were conducted.

\subsection{Results}

Shown in Fig. 4 are the effects of varying the ISI on the redundant, background and figure conditions as measured by DR. ISI had a monotonic effect on performance for conditions where the background component of the stimuli changed. As in Experiment 1 , discrimination of the figure condition was poor. A repeated measures ANOVA (condition $\times$ ISI) confirmed this significant interaction, $F(14,42)=14.0$. Subsequent analyses looking at each condition in isolation found significant main effects of ISI on DR for both the background and redundant conditions, $F \mathrm{~s}(7,21)>18.1$, but no significant effect of ISI for the figure condition. A series of single mean $t$-tests looking at the combined redundant and background condition performance for each individual bird revealed that all ISI values supported significantly above-chance discrimination out to $1000 \mathrm{~ms}$ for each pigeon, and two birds, \#3P and \#4R, were significantly above chance at ISIs of $2000 \mathrm{~ms}$ $(t \mathrm{~s}(4)>3.1)$.

This drop in performance with increasing ISI occurred for both same and different sequential information. The finding is indicated by the concurrent changes in peck rate over ISI for both types of sequences. Mean peck rates for same sequences significantly decreased with increasing ISI $(22.8,22.1,22.7$, $18.9,20.1,18.2,16.5,13.4$ for each ISI, respectively; $F(7,21)=9.9)$. Correspondingly, mean peck rates for redundant and background different sequences significantly increased with increasing ISI $(8.1,10.2$, $10.2,12.3,13.1,13.3,13.9,13.8$ for each ISI, respectively; $F(7,21)=7.4)$. Finally, we examined the effect of ISI on responding to just the second item of a sequence in analyses similar to Experiment 1. This analysis effectively isolates any ISI effect to just a single interval. Results based on responding to just the second item were the same as measured over the 


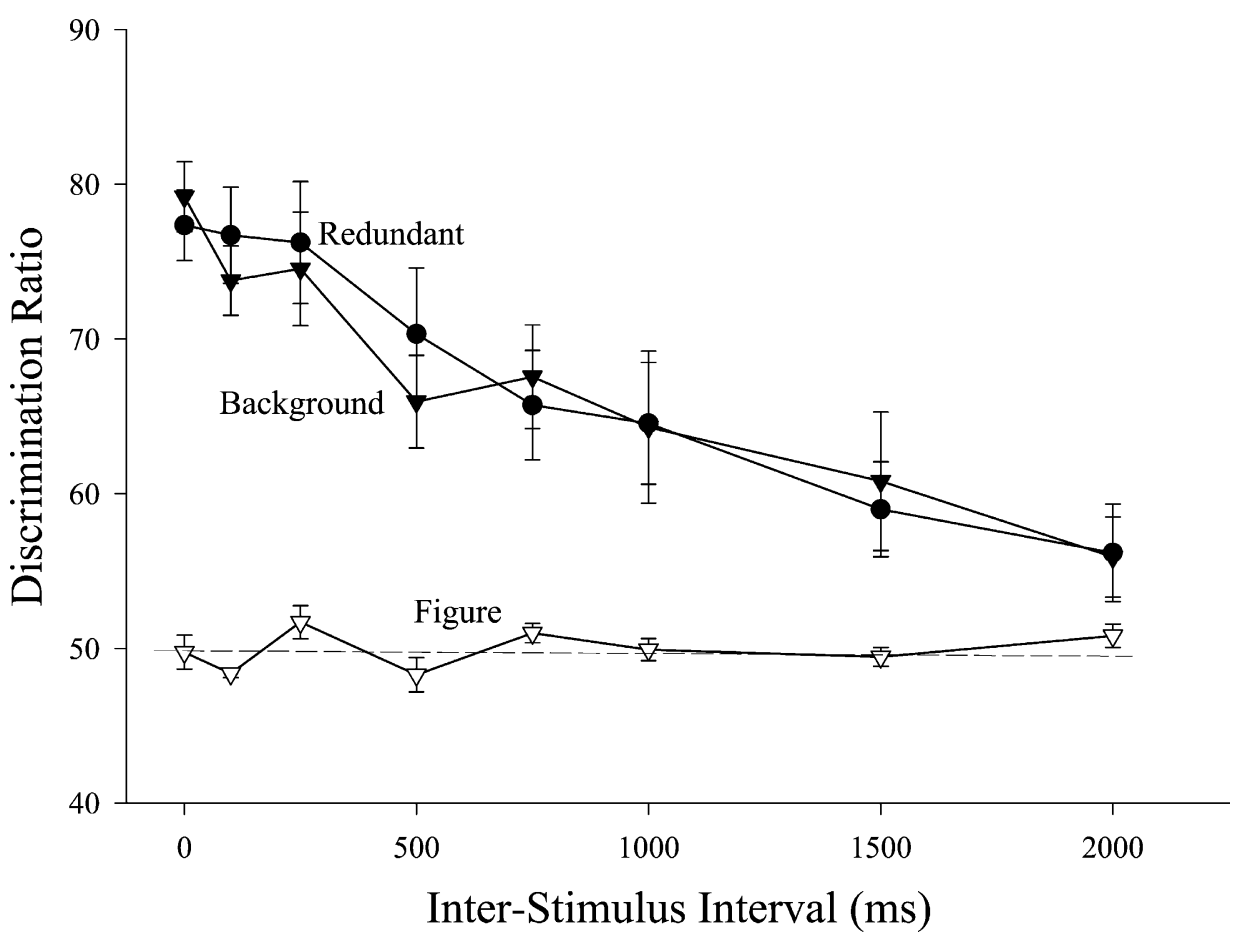

Fig. 4. Mean discrimination ratio and standard errors for the redundant, background and figure conditions over the eight ISIs tested in Experiment 2. The dashed line represents chance discrimination.

entire presentation interval. Again excluding the figure condition trials, mean DRs based on peck counts to just the second item were $71,67,65,62,63,64,59$, and 54 for each ISI, respectively.

\subsection{Discussion}

Overall, increasing the ISI monotonically decreased the pigeons' discrimination on both same and different sequences. This result suggests that some form of short-term item memory was involved in mediating the discrimination. All of the birds tolerated at least $1 \mathrm{~s}$ between items and two tolerated a temporal separation of $2 \mathrm{~s}$. In general, the pattern of ISI results observed here are very similar to those obtained by Young et al. (1999) when they manipulated ISI in their multiple-item successive procedure. They found that pigeons could likewise discriminate at above chance levels with ISIs of $2 \mathrm{~s}$ or less, although at longer ISIs their pigeons started showing an overall bias to report "same" to both kinds of sequences.
Together, both studies suggest that pigeons can bridge temporal gaps of about $2 \mathrm{~s}$ between successively presented items. This indicates that memory for information derived from the previous items must be involved, so that increasing the ISI acts as a memory delay reducing their ability to retain how the prior items might be similar or different from the one currently in view (see Killeen, 2001 for a potential model for how this short-term memory might function). In general, these values are smaller than the $2-8 \mathrm{~s}$ delays that pigeons can frequently tolerate in matching-to-sample tasks. One possible reason for this difference is that in the S/D task, the encoding of the prior item needs to be retrieved during the presence of the next stimulus. When the next stimulus matches the prior one, retrieval of the prior information is easy, but when it is different, the context has changed, and retrieval of it may be more difficult (Tulving, 1983; White, 2002). In the MTS context, one of the test stimuli always matches the sample and thus consistently helps to retrieve the prior sample information on each trial. 


\section{Experiment 3}

The objective of Experiment 3 was to see if the pigeons would transfer the S/D discrimination to novel picture items. Successful transfer would indicate that the discrimination was not stimulus-specific, and suggest instead that it was done by detecting the successive relation between two stimuli. Two transfer tests were conducted. The first examined transfer to color photographs of natural objects and scenes. The second examined transfer to gray-scale photographs of comparable content. All involved non-reinforced presentations of same and different sequences comprised exclusively of novel photographs.

Five separate subtests were conducted examining transfer to color photographs. Each subtest tested novel combinations of six novel photographs. Following each subtest, these six stimuli were integrated into the pigeons' daily baseline testing. Concurrently, the number of trials testing the figure and background stimuli from Experiments 1 and 2 was reduced. By the start of the fifth and last color transfer test, all testing of these older stimulus items was discontinued, and the pigeons were exclusively discriminating randomized combinations of the 24 pictures used in subtests one to four. The gray-scale tests followed these color tests. The gray-scale tests consisted of two subtests. The first subtest looked at the transfer to gray-scale versions of familiar color photographs. The second subtest looked at transfer with completely novel gray-scale pictures. These gray-scale tests allowed us to assess the role of color, shape, and luminance cues in the discrimination.

\subsection{Method}

\subsubsection{Animals and apparatus}

The same pigeons and apparatus were used as in Experiments 1 and 2.

\subsubsection{Color transfer tests}

The pigeons were tested in a series of five independent tests. Each consisted of two sessions involving testing same and different sequences of novel pictures as non-reinforced probe tests (no reinforcement or timeouts). Each transfer session consisted of 12 test trials (6 same/6 different) randomly mixed (after the 20th trial) into a session's scheduled baseline trials.
For all trials, the stimulus duration per picture was $2000 \mathrm{~ms}$ and each lasted $20 \mathrm{~s}$. Half of the transfer trials were tested with $100 \mathrm{~ms}$ ISI and half with a $500 \mathrm{~ms}$ ISI. Baseline trials were tested with equal numbers of four ISIs $(0,100,500,1000)$ within a session. Twenty-five percent of the baseline same trials were conducted as probes to measure pecking behavior.

The six different color transfer trials in each session tested unique combinations of the six novel pictures (randomly selected real-world objects and scenes) used in each subtest, with each picture tested once in the even and odd ordinal positions of a sequence. The six same color transfer trials were unique in the first session and simply repeated in the second test session. Following the two test sessions, the six transfer stimuli were then integrated into the pigeons' daily testing, and the number of trials using Experiment 1's background and figure stimuli simultaneously reduced. With the integration of additional subtests, slight adjustments in the number of trials per session were made to maintain counterbalancing, while gradually eliminating the older stimuli. By the fifth transfer test, the pigeons were being tested exclusively with photographic stimuli from the four previous subtests. Approximately 10 sessions separated the five color transfer tests.

\subsubsection{Gray-scale transfer tests}

Each transfer session contained 12 trials (6 same/6 different) with either familiar (first four sessions) or novel gray-scale (next four sessions) novel pictures tested as non-reinforced probe tests. These probe tests were randomly mixed (after the 20th trial) into a session's scheduled baseline trials. Transfer trials were conducted using a $100 \mathrm{~ms}$ ISI and a viewing duration of $2000 \mathrm{~ms}$ per item and lasted for $20 \mathrm{~s}$. A total of 12 familiar and 12 novel, 256-level, gray-scale photographs were tested over eight sessions. The first block of four sessions tested familiar pictures and the second block tested novel pictures. Different trials (six per session) involved unique combinations of these pictures, with each appearing once in the even or odd ordinal position of one of the sequences. The same sequences were trial-unique for the first two sessions (six per session), and repeated once more in the last two sessions of a block. The baseline trials were randomly constructed from the pool of 30 photographic stimuli. 


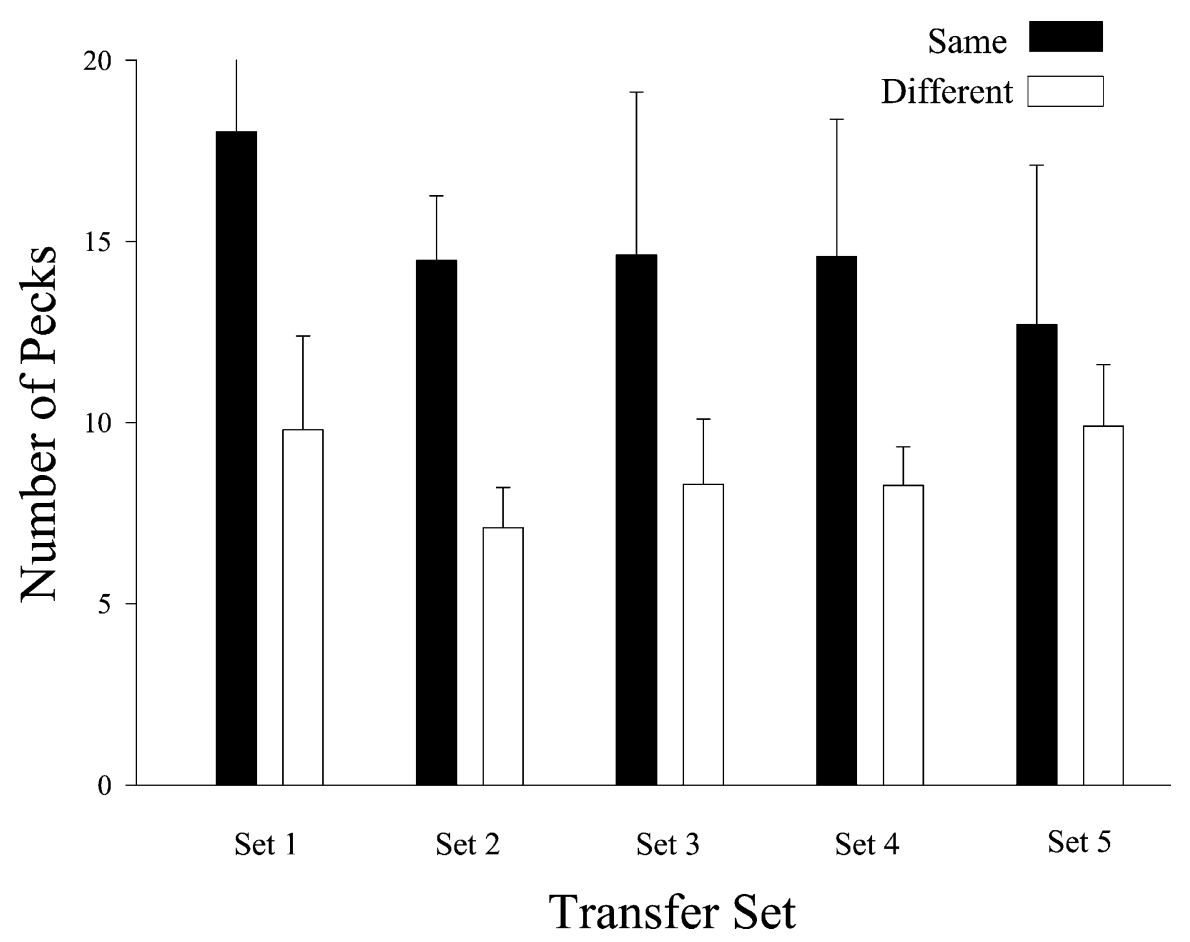

Fig. 5. Mean number of pecks and standard errors to same and different sequences created from novel color photographs during the five transfer tests in Experiment 3.

\subsection{Results}

\subsubsection{Color transfer tests}

The pigeons transferred to the novel color stimuli (see Fig. 5). This figure shows that peck rate was consistently higher on novel same trials than on different trials across all five tests. Combined across all tests, same trial peck rates were significantly higher in the $100 \mathrm{~ms}$ ISI ( same $=15.1$ pecks; different $=9.2$ ) and 500 ISI condition ( same $=14.2$ pecks; different $=$ 9.8). An ANOVA (Test Block $\times$ ISI $\times$ same/different) found a significant difference between peck rates on novel same and different trials, $F(1,3)=31.5$, but no effects of Test Block, ISI, or their interaction with this factor. With the subsequent introduction of the prior transfer items into the daily baseline trials, the discrimination of these former transfer items improved with testing and differential reinforcement. By the fifth test, for example, peck rates on same trials (24.1 pecks) was greater than on different trials (8.1 pecks) for such trials. The consistently lower peck rates during transfer testing indicate the pigeons were detecting the novelty of the unfamiliar transfer stimuli.

As in Experiment 1, we examined the pigeons' transfer behavior over successive presentations within a trial. Fig. 6 shows the mean peck counts during each presentation for the novel color same and different sequences. Since there was no significant effect of ISI on transfer, these data were combined for this analysis. As with the baseline items, novel same and different transfer sequences were being discriminated by the second item, $F(1,3)=23.5$. Unlike the baseline results in Experiment 1, there was little growth in the discrimination with additional transitions after 2000 or $3000 \mathrm{~ms}$. Finally, note that most of the changes in pecking behavior were associated with increased peck rates to the same sequences for the transfer trials, whereas in Experiment 1 the primary change was associated with a decrease in peck rate to the different sequences (cf. Fig. 3). 


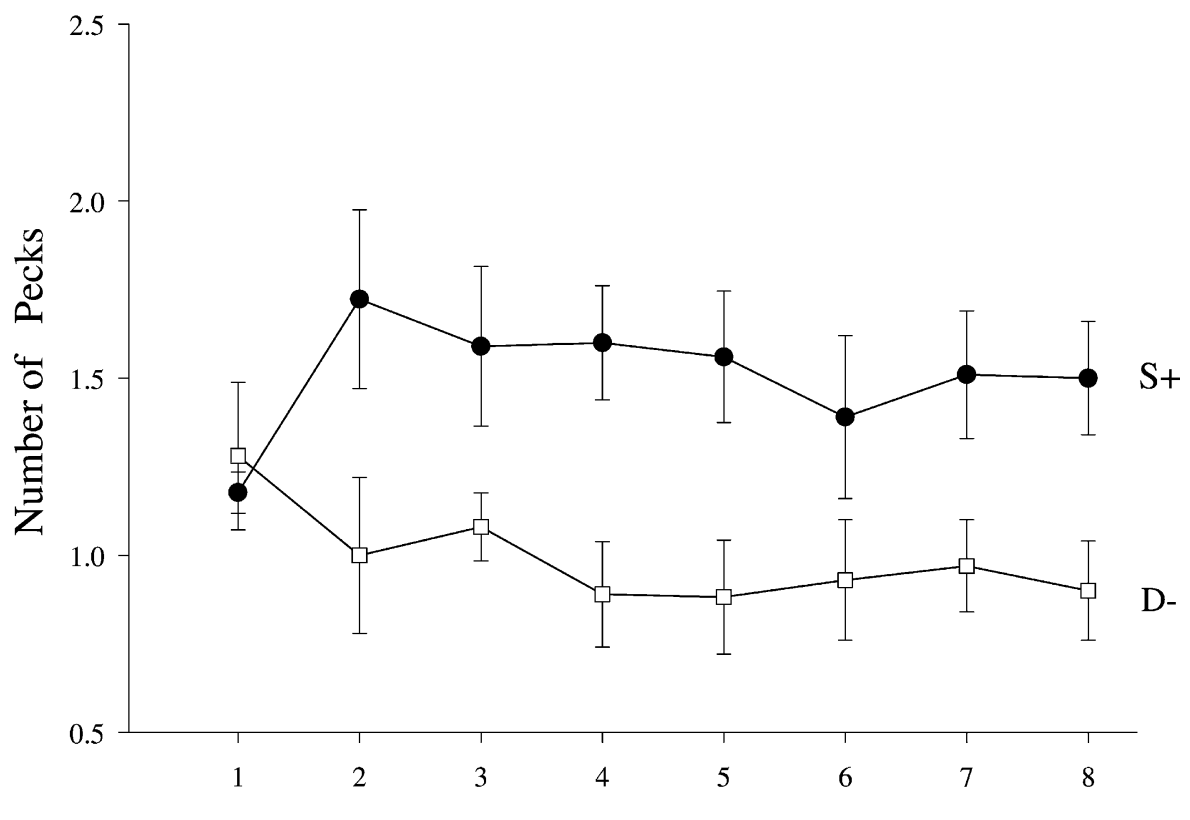

Successive $2000 \mathrm{~ms}$ Presentation Intervals

Fig. 6. Mean number of pecks and standard errors for all four birds across successive intervals for novel same and different color transfer trials in Experiment 3.

\subsubsection{Gray-scale transfer tests}

Discrimination transfer occurred with the novel gray-scale pictures, but took longer to emerge within a trial than with the color stimuli. Fig. 7 shows mean peck counts across presentations for novel gray-scale same and different sequences. Unlike color testing, it took several transitions for the peck rates to same and different sequences to diverge. Because of this, we divided the presentation interval into four 5-s bins, which revealed that transfer performance was indeed best during the last $5 \mathrm{~s}$ of the interval. Examination of transfer performance during the last 5 -s bin revealed that three of the four birds significantly transferred their discrimination to the novel gray-scale photographs. Individual tests revealed that \#1B, \#3P and \#4R each showed significant differences in peck rates between the novel different (mean $=2.4$ pecks) and same sequences (mean $=$ 5.5 pecks; $\mathrm{DR}=69.6$; all $F \mathrm{~s}(1,3)>10.1)$. The fourth bird, $\# 2 \mathrm{~N}$, showed no significant difference in peck rates to the same (4.2 pecks) and different sequences (4.5 pecks). For comparison, peck rates for the baseline stimuli over the last 5-s interval revealed significant differences in peck rates to each sequence (\#1B, \#3P and \#4R combined: $\mathrm{S}+=6.0$ pecks, $\mathrm{D}-=2.2$ pecks; $\mathrm{DR}=73.1$, as did bird $\# 2 \mathrm{~N}$ as well, $\mathrm{S}+=5.1$ pecks, $\mathrm{D}-=2.5$ pecks; $\mathrm{DR}=67.1$ ).

Analyses of transfer using the gray-scale versions of familiar color pictures revealed a slightly different story. Unlike with the novel items, all the pigeons transferred to the familiar gray-scale items and did so by the second item presentation within a sequence. In addition, overall peck rates were higher with the familiar gray-scale displays than with the novel ones. Combined across all four sessions, peck rates were significantly higher on novel same sequences (mean $=$ 22.9) than on novel different sequences (mean $=13.9$; $\mathrm{DR}=62.2 ; F(1,3)=20.7)$. There was no effect of session or its interaction with trial type. Further analyses looked at peck rates to same and different sequences during second item presentation. These revealed a significantly higher peck rate on novel same sequences (mean $=2.8$ ) than on novel different sequences $($ mean $=1.7 ; F(1,3)=10.4)$. The discrimination across subsequent intervals looked similar to that depicted in Fig. 6 for the color transfer tests. Finally, we found a significant difference in overall 


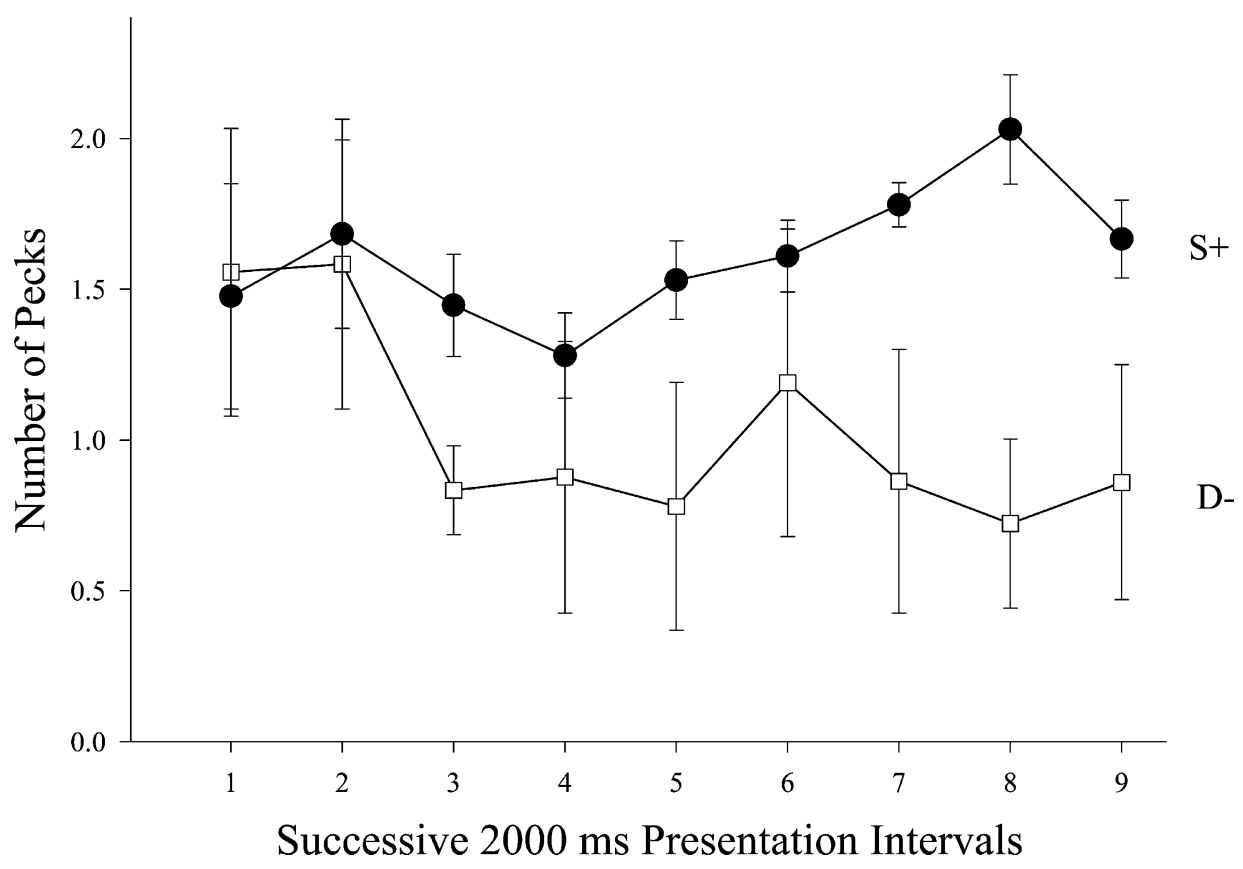

Fig. 7. Mean number of pecks and standard errors across successive intervals for novel same and different gray-scale transfer trials in Experiment 3.

peck rate on same sequences for novel (mean $=$ 12.3) and familiar gray-scale stimuli (mean $=19.1$, $F(1,3)=12.1)$ in a direct comparison across the two separate tests.

\subsection{Discussion}

The most important finding of Experiment 3 was the significant above-chance transfer to novel stimuli. All four pigeons transferred to the novel color pictures and novel gray-scale pictures derived from familiar color pictures, and three of the four pigeons transferred to completely novel gray-scale pictures. For color stimuli and "familiar" gray- scale pictures, evidence of discrimination emerged by the second item presentation, but control by the novel gray-scale stimuli took until the third item to emerge and was best during the last part of the presentation interval. These transfer results rule out the possibility that the discrimination was based on learning stimulus-specific associations (e.g. Carter and Werner, 1978; Wright, 1997, 2001). Instead, the positive transfer suggests the pigeons were responding to the general stimulus relations of same and different between successive items.
Their success with the gray-scale stimuli indicates that the discrimination was not based exclusively on color cues, but must have involved recognizing differences in shape or brightness as well. One revealing fact is that the familiar gray-scale images supported higher overall peck rates than did the novel gray-scale pictures. This finding strongly suggests the pigeons were recognizing non-colored features of the familiar color pictures within these stimuli. These features potentially include the shape of the objects within the images, but at least must consist of the relative patterns of light and dark contrasts common to both formats. If so, this interpretation suggests the pigeons were using more than just gross perceptual changes to perform the discrimination, but were remembering and comparing specific properties and patterns across successive items.

\section{Experiment 4}

The objective of Experiment 4 was to see if the pigeons would transfer their discrimination to video stimuli. These video stimuli allowed us to 
examine how the pigeons reacted to constantly different and changing stimuli. If they could continue to discriminate them, it would argue against a simple perceptual change hypothesis as the basis for this discrimination, since there would be continuously transforming shape, color, and brightness changes at all times within and between the items of a video sequence. In addition, the development of a testing procedure for looking at how animals perceive and discriminate video stimuli per se would be of considerable value given the growing interest in such stimuli for studying animal behavior (e.g. Adret, 1997; McQuoid and Galef, 1993; Patterson-Kane et al., 1997; Shimizu, 1998).

The videos consisted of 2-s segments captured from various movies. Testing was done in two phases. The first phase consisted of two sessions testing four videos in a probe transfer design. Because two of three pigeons were very reluctant to peck the video stimuli, we discontinued this test. The second phase tested the processing of the video stimuli using a reinforcement-based design. In this phase, the video stimuli were presented in the standard same or different sequences with regards to their imaged content, but we also manipulated the serial ordering of the individual frames within these videos as well. Two types of frame orderings were examined. The individual frames of the video stimuli could either be presented in their normal coherent order (forwards or backwards) or as a one of two randomized sequences of frames that broke up the moving continuity of the imaged content. Thus, when tested on a "same" content trial, we could examine whether either of these different types of sequences themselves were sufficient to generate a "different" response. Because these videos were smaller in size than the previously tested static pictures, a period of training with reduced-sized static pictures was done prior to video testing.

\subsection{Method}

\subsubsection{Animals and apparatus}

The same pigeons and apparatus were again used. One bird (\#1B) was temporarily not tested because of health reasons.

\subsection{Procedure}

\subsubsection{Reduced-sized transfer and training}

To test for transfer to reduced-sized static stimuli, two sessions testing same and different sequences of familiar pictures at smaller size $(352 \times 240$ pixels) were conducted. Each session consisted of 10 trial-unique reduced-size probe test trials (5 same/5 different, no reinforcement) added to each session using a transfer design identical to Experiment 3's. All three pigeons transferred their discrimination to the smaller stimuli (mean DR $=78.8$ ). A repeated measures ANOVA (trial type $\times$ size) revealed a significant difference in peck rate to the same and different sequences, $F(1,2)=19.89$, with no interaction across the large and small stimulus size. There was a significant main effect of size, $F(1,2)=19.87$, as the pigeons pecked less frequently at the smaller stimuli (mean number of pecks $=13.6$ ) than the large ones (17.8).

After this test, the reduced size stimuli were added to the daily mixture of differentially reinforced trials, with their numbers gradually increased over 20 sessions. In the five sessions prior to video testing, the pigeons experienced 128-trial (64 same/64 different trials) baseline sessions constructed from a pool of 56 color photographs (due to further additions made after Experiment 3) of two different sizes (sizes were not mixed). These trials lasted $20 \mathrm{~s}$, used a $2000 \mathrm{~ms}$ viewing duration per item and tested equal numbers of four ISIs $(100,500,750,1000 \mathrm{~ms})$ within a session.

\subsubsection{Video tests}

Two tests were performed with video stimuli. Each test involved the successive presentation of 2000-ms segments of video captured from different movies. The nine videos were $352 \times 240$ pixels in size and depicted different events (helicopter flying, rafting downstream, car accelerating, caribou fighting, bird flying, dinosaurs chasing people, horse race, overhead plane flight, hang gliding off cliff).

The first test consisted of two sessions. In each session, eight non-reinforced probe trials using video stimuli and eight probe trials using reduced size stimuli were randomly mixed among the baseline trials (each type testing 4 same/4 different made from combinations of four stimuli). These transfer trials last $20 \mathrm{~s}$ 
Table 1

The eight video conditions from the second test of Experiment 4

\begin{tabular}{|c|c|c|c|}
\hline & Video content & $\begin{array}{l}\text { Order of } \\
\text { frames }\end{array}$ & $\begin{array}{l}\text { Type of frame } \\
\text { ordering }\end{array}$ \\
\hline DDC & Different & Different & Coherent $^{\mathrm{a}}$ \\
\hline DDR & Different & Different & Randomized $^{\mathrm{b}}$ \\
\hline DSC & Different & Same & Coherent \\
\hline DSR & Different & Same & Randomized \\
\hline SDC & Same & Different & Coherent \\
\hline SDR & Same & Different & Randomized \\
\hline SSC & Same & Same & Coherent $^{\mathrm{c}}$ \\
\hline SSR & Same & Same & Randomized $^{\mathrm{c}}$ \\
\hline
\end{tabular}

${ }^{\text {a }}$ Coherent videos were presented either in a normal forward or backward ordering within a trial.

${ }^{b}$ Randomized videos were presented using either one of two fixed randomized orderings, making them comparable to the two sequences in the coherent conditions.

$\mathrm{c}$ These two conditions were the only ones reinforced, since there was no ambiguity as to whether they should be treated as same trials.

and were conducted using a 100-ms ISI and a 2000-ms viewing duration.

The second test consisted of 20 112-trial sessions. Sixty-four of these were baseline trials that tested equal numbers of large or reduced-sized static pictures at one of four different ISIs $(0,100,500,1000)$. The remaining 48 trials tested eight combinations of video stimuli (see Table 1). Four of these conditions successively alternated videos identical in their visual content, but which varied in the sequential ordering of the video's separate frames. These conditions were: (1) the frames of the two videos were presented in a coherent forward or backward order (SSC), (2) the frames of the two videos were presented in one of two randomized orders (SSR), (3) the frames of successive videos alternated between streaming forward and then backward (SDC), (4) the frames of successive videos alternated between the two randomized sequences (SDR). The four other conditions successively alternated videos that were different in their content along with the nature of their ordering of the frames. These conditions were: (5) the frames of both were presented in either a coherent forward or backward order (DSC), (6) the frames of both videos were presented in the same one of two randomized orders (DSR), (7) the frames of both videos alternated between streaming forward and backward (DDC), (8) the frames of both videos alternated between the two randomized sequences (DDR). These eight video con- ditions were tested six times within a session. Only the coherent and randomized videos sequences in which both the content and frame orders were identical were reinforced, with one-third of these trials conducted as probe trials.

\subsection{Results}

Overall the pigeons did poorly during the initial two transfer sessions with video stimuli. The primary reason for this was that two of the three pigeons pecked infrequently at the video sequences (mean pecks to video stimuli, \#3P =0; \#4R = 1.5). The third pigeon did peck at the video sequences and showed a difference in peck rate to the same sequences (mean $=12.2$ ) over the different sequences (mean $=6.6$ pecks), but a substantial number of trials with no pecks as well made this difference statistically unreliable.

The second test with coherent and randomized video stimuli revealed that the pigeons could discriminate same and different video sequences from one another and that discrimination was based on content changes between the videos, but not the ordering of the frames within the videos. Shown in Fig. 8 are the results from the eight video conditions tested in the second phase. These data show that the pigeons were rapidly able to discriminate video sequences (in one or two sessions) where the content of the videos changed across successive intervals from when the same videos were repeated. Over the last 10 sessions, the two conditions in which the same video and frame order was successively repeated (SSC; SSR) supported significantly higher peck rates in each pigeon than did the different sequences (DDC; DDR), all $F \mathrm{~s}(1,9)>5.12$. It made no difference within these two boarder categories, however, whether the videos were presented in a smoothly changing coherent manner or a randomly-ordered fashion. The pigeons showed no sensitivity to the ordering of the videos. That is, statistical comparisons of the conditions in which frame ordering was the only difference between the successive presentations of identical content (SDC; SDR) did not significantly differ from the other same conditions (SSC; SSR), indicating that frame order was not a sufficient basis for the pigeons to discriminate successive videos. One consistent difference between responding on video and static trials was that overall peck rates were generally lower to 


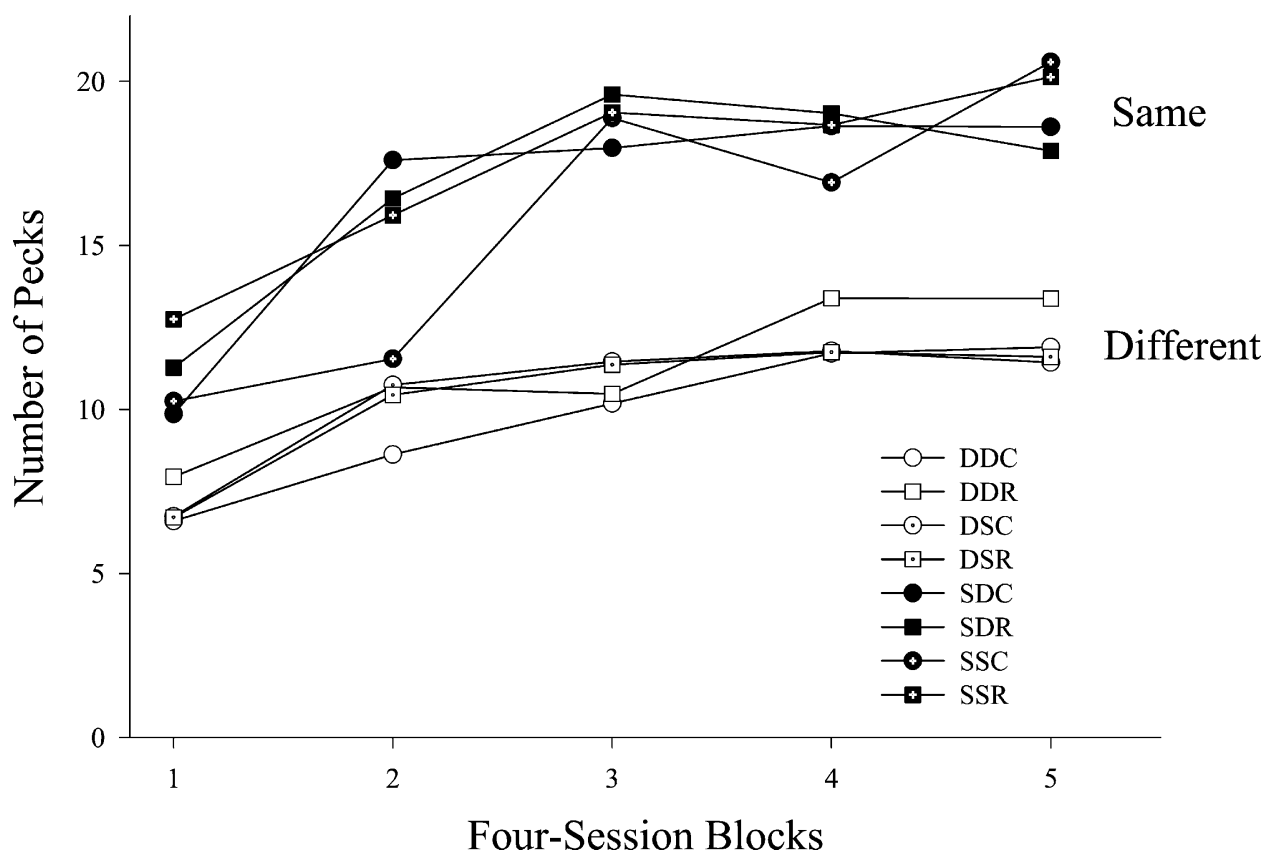

Fig. 8. Mean number of pecks in the eight video conditions tested in Experiment 4. The three letter code denotes first whether the videos were same (S) or different (D) across intervals, second whether the order of the frames were same (S) or different (D) across intervals, and third whether this ordering was coherent $(\mathrm{C})$ or randomized (R). See Table 1.

the videos. A comparison of peck rates to same sequences on video trials over the last eight sessions (mean $=19.0)$ was significantly lower than either the reduced size (mean $=30.5$ ) or large static pictures $($ mean $=28.9)$.

\subsection{Discussion}

These results indicate that pigeons can discriminate the identity and difference of successively alternating video stimuli, at least when differentially reinforced. In the initial non-reinforced transfer test, we found the pigeons were very reluctant to peck the video stimuli at first, perhaps because of neophobia in response to their extended history with static stimuli.

There was no evidence, however, that the pigeons were processing the specific ordering of the frames comprising the videos. Presenting them in a natural and smoothly changing manner or as a more unnatural, randomly-ordered fashion made no difference. While we had anticipated the trouble with distinguishing the ordering of the two randomized sequences (random sequence 1 versus random sequence 2), we had thought the forward versus backward ordering of the coherent videos might be discriminable. In a prior study, Cook et al. (2001) tested coherent and randomly-organized videos of an action-based sequence discrimination. This discrimination required the pigeons to tell whether a camera's viewpoint went around or through the central opening of an approaching object (examples of these videos can be seen on-line at the Animal Cognition journal website). They found that coherent videos supported better discrimination than did randomized one. We had thought a similar effect might occur here, but clearly did not (see Jitsumori et al., 1999 for a similar result involving forward and backward videos). Such results indicate that the motion within the video's content was not critical to the pigeons. As such, could the pigeons have been simply seeing the videos as static displays? Dittrich and Lea (1993) found that pigeons can easily discriminate between static pictures and dynamic videos of similar content, so it is likely they were doing so here. Further, the reduced peck rates to the videos and the gradual improvement with experience each suggest that the videos were seen as 
fundamentally different from the static displays. In fact, one potential reason that peck rates were generally lower is that they were reacting to the constant local "difference" between successive frames within a single video.

Why the difference between this study and Cook et al.'s (2001) experiments regarding the effect of frame randomization on the pigeons' reactions to these video stimuli? One important factor may have been the pigeons' extensive training with static images prior to the video test. This factor may have resulted in the pigeons learning to pay more attention to static color and shape cues in comparison to the previously non-existent motion cues. Cook et al.'s pigeons were trained from the outset with motion being a relevant cue. Given their prior static training and absence of a compelling need to process motion prior to the current manipulation, perhaps it is not so surprising the current pigeons did not do so. The use of videos that specifically require attention to the video's movement within a S/D discrimination clearly need to be tested. Nevertheless, the fact that the pigeons could perform the task with these ever-changing stimuli further argues against any simple perceptual change hypothesis as the basis for their S/D discrimination.

\subsection{General discussion}

These experiments provide evidence that pigeons can differentiate same and different sequences based on the alternation of only two picture stimuli. We found they can discriminate both moderate to large pictorial changes in color, gray-scale, and video stimuli, although stimuli with smaller differences proved difficult for the pigeons to learn. This discriminative behavior transferred to both novel color and gray-scale static pictures and showed savings in tests using video stimuli. Further, the discrimination was maintained when tested with large numbers ( $>55$ pictures) of randomly combined photographic stimuli of two different sizes. Except in the case of novel gray-scale pictures during transfer, the same/different relation within any sequence was detected by the second item. Additional item transitions further improved performance suggesting the involvement of an accumulative memory-based comparison process in this discrimination. The monotonic effects of increasing ISI on performance further suggest such a memory process.
These data suggest pigeons can learn to discriminate S/D relations without the necessity of simultaneous presentation, and when combined with our previous simultaneous results, suggests they can conceptually learn to discriminate S/D relations across a variety of temporal and spatial arrangements.

The successful learning and transfer across sequences of successively presented visual stimuli helps to eliminate several alternative interpretations of our earlier S/D results (Cook et al., 1995, 1997, 1999) collected using a simultaneous procedure. For instance, the results argue against concerns that our previous S/D results were due to the "generalized" detection of perceptual patterns or spatial anomalies within the mosaic of repeated elements in those displays. If the pigeons were only capable of using such simultaneous patterns to guide their S/D choices, then the successive nature of the present task should have made use of those cues impossible. Element simultaneity apparently is not a necessary condition for pigeons to learn to discriminate S/D relations.

Besides prevailing against perceptual alternatives, these results speak to two other issues with our simultaneous procedure. The first involves the possibility that differential responding to the same and different displays was critical to the performance of the discrimination. In our simultaneous procedure, the pigeons were required to localize and peck the odd element in the different displays. The number of pecks to accomplish this was then yoked to comparable same trials, where there were no odd "targets". While this yoking procedure equated the number of pecks between trial types, the different displays did require a series of localized pecks not required of the same displays. The present results indicate that such observing behaviors are not necessary for learning about S/D relations.

The second issue involves the number of elements in the displays. We have consistently used multiple items in create our simultaneous S/D displays. In humans, chimpanzees, and monkeys (e.g. Katz et al., 2002; Premack and Premack, 1983), S/D judgments can easily be generated from the comparison of only two stimuli. However, such S/D judgments have been difficult to attain in avian species without extensive training (Pepperberg, 1987; Santiago and Wright, 1984). The present results make the case that pigeons, too, can make S/D discriminations based on the successive comparison of only two items. While multiple 
alternations of the two items were employed during training, the pigeons eventually required only one transition to begin responding differentially to same and different sequences. We cannot judge whether these extended sequences were critical, however, in the initial acquisition of the discrimination. These repeated transitions did provide further information as their discriminative behavior improved for several transitions beyond the first one. This finding contrasts with that of Young et al. (1999) who found that number of transitions in their sequences was not critical to their pigeons' choice behavior when tested with small numbers of stimuli.

Overall, the current results are consistent with the hypothesis that pigeons can learn to process the qualitative S/D relations between various stimuli whether presented at the same time or over time. The transfer across color and gray-scale static images and the rapid acquisition with video stimuli suggest that a non-stimulus-specific relational account is required. As always, the debate is whether these studies require a conceptual account or can be explained by lower-level perceptual alternatives.

What was the basis for this determination of pictorial sameness and difference in the current study? While the color in the pictures likely played a key role, Experiment 3 showed that gray-scale static pictures are fully capable of supporting discrimination and transfer without prior experience. Thus, the pigeons' were detecting feature changes other than color across pictures. As discussed in Experiment 3, the overall greater responsiveness and discrimination of the familiar gray-scale pictures by the pigeons suggests they were recognizing them based on their prior experience with the colored versions of these same images. Such data suggest the pigeons were at least sensitive to the pattern of light and dark areas across the images, and potentially to the shape of the objects depicted within them.

The other critical factor in these experiments was the amount of change between displays. Over the four experiments, the pigeons had little problem with displays in which moderate to large changes occurred between successive items, but Experiments 1 and 2, showed that small, centrally located changes were difficult for the birds. Young et al. $(1997,1999)$ found that pigeons could successfully discriminate very small successively presented icon stimuli, but in their case a large number of items were presented in each sequence and each item was pecked. In humans, detecting certain kinds of changes or difference between successively presented pictures seems to require focused attention to be specifically brought to the area of the display that is changing (e.g. Rensink et al., 1997). In fact, very large changes can often go unnoticed by people in scenes without such selection (Simons and Levin, 1998). Similar acts of attention may also be required in other animals to detect change across time. So far, for example, pigeons have only been successful in successive S/D discriminations where the entire stimulus changed from one interval to the next. This type of complete change essentially eliminates the need for attention or search for any specific place or feature within an image. Exactly how attention and the degree of change between displays are involved in the present discrimination needs further exploration to understand how pigeons compare successive pictures for their identity and difference.

In addition to comparing color and pattern information between static pictures, the current studies revealed that the pigeons could perform this discrimination with video stimuli. While the pigeons reacted poorly upon the introduction of these stimuli, all of the pigeons differentiated these stimuli in just a few sessions. Despite the ever-changing local nature of these stimuli, the pigeons could still extract sufficient information to discriminate same or different video sequences. What did not matter is whether the videos were coherently or randomly scrambled in terms of the presentation. This finding suggests they were not processing the sequential order of the information within a video, and perhaps not even seeing them as coherent presentations of the real world. Pigeons appear to have a higher threshold for distinguishing coherent from random motion (Bischof et al., 1999) and this may have played a role in limiting their capabilities to distinguish the different modes of video presentation tested here.

Given all of these considerations, it seems most likely the pigeons were comparing image-based memories of the successive stimuli across time, and were not just responding to, say, the changing luminance of successive pictures. The gray-scale recognition of familiar colored pictures and their capacity to deal with the constantly fluctuating video stimuli suggest that such a simple perceptual hypothesis is not adequate. 
Given this, we remain comfortable in suggesting a conceptual alternative as the basis for our pigeons' ability to discriminate the wide variety and growing number of S/D configurations that have been tested. So far in every test, where we have tried to evaluate or eliminate perceptual alternatives (current study; Cook et al., 1997, see also Young and Wasserman, 2001) little support for such proposals has been garnered. Even ignoring this collected evidence, we would still be left with the hypothesis that the pigeons are using different sets of perpetual cues to solve the same underlying conceptual task.

As such, a key direction for future research will be to compare directly the nature of the stimulus relations between successive and simultaneous discrimination procedures. There is now a large body of evidence that pigeons can judge stimulus relations presented simultaneously, and a growing one indicating that this capacity extends to successive displays. Together, they form a nice set of converging evidence that visual relations between elements can control pigeon behavior. What is missing is direct empirical evidence for the assumption that the same processes are involved in both procedures. If separate "perceptual" solutions lie at the heart of these different procedures than the transfer between them should be minimal. If, on the other hand, the pigeons are sensitive to stimulus relations in either setting, then any "conceptual" discrimination learned in one procedure should transfer to the other procedure.

\section{Acknowledgements}

This research was supported by a grant from the National Science Foundation to R.G. Cook.

\section{References}

Adret, P., 1997. Discrimination of video images by zebra finches (Taeniopygia guttata): direct evidence from song performance. J. Comp. Psychol. 111, 115-125.

Bischof, W.F., Reid, S.L., Wylie, D.R.W., Spetch, M.L., 1999. Perception of coherent motion in random dot displays by pigeons and humans. Percept. Psychophys. 61, 10891101.

Bovet, D., Vauclair, J., 2001. Judgment of conceptual identity in monkeys. Psychon. Bull. Rev. 8, 470-475.
Carter, D.E., Werner, J.T., 1978. Complex learning and information processing in pigeons: a critical analysis. J. Exp. Anal. Behav. 29, 565-601.

Cook, R.G., 2001. Avian Visual Cognition [On-line]. Available: http://www.pigeon.psy.tufts.edu/avc/.

Cook, R.G., 2002a Same/different learning in pigeons. In: Bekoff, M., Allen, C., Burghardt, G. (Eds.), The Cognitive Animal. MIT Press, Cambridge, MA, pp. 229-238.

Cook, R.G., 2002b. The structure of pigeon multiple-class same/ different learning. J. Exp. Anal. Behav. 78, 345-364.

Cook, R.G., Wixted, J.T., 1997. Same-different texture discrimination in pigeons: testing competing models of discrimination and stimulus integration. J. Exp. Psychol. Anim. Behav. Process. 23, 401-416.

Cook, R.G., Cavoto, K.K., Cavoto, B.R., 1995. Same/different texture discrimination and concept learning in pigeons. J. Exp. Psychol. Anim. Behav. Process. 21, 253-260.

Cook, R.G., Katz, J.S., Cavoto, B.R., 1997. Pigeon same-different concept learning with multiple stimulus classes. J. Exp. Psychol. Anim. Behav. Process. 23, 417-433.

Cook, R.G., Katz, J.S., Kelly, D.M., 1999. Pictorial same-different categorical learning and discrimination in pigeons. Cahiers de Psychologie Cogn. 18, 805-844.

Cook, R.G., Shaw, R., Blaisdell, A.P., 2001. Dynamic object perception by pigeons: discrimination of action in video presentations. Anim. Cogn. 4, 137-146.

Dittrich, W.H., Lea, S.E.G., 1993. Motion as a natural category for pigeons: generalization and a feature-positive effect. J. Exp. Anal. Behav. 59, 115.

Jitsumori, M., Natori, M., Okuyama, K., 1999. Recognition of moving video images of conspecifics by pigeons: effects of individuals, static and dynamic motion cues and movement. Anim. Learn. Behav. 27, 303-315.

Katz, J.S., Wright, A.A., Bachevalier, J., 2002. Mechanisms of same/different abstract-concept learning by rhesus monkeys (Macaca mulatta). J. Exp. Psychol. Anim. Behav. Process. 28, 358-368.

Killeen, P.R., 2001. Writing and overwriting short-term memory. Psychon. Bull. Rev. 8, 18-43.

Mackintosh, N.J., 2000. Abstraction and discrimination. In: Heyes, C., Huber, L. (Eds.), The Evolution of Cognition. MIT Press, Cambridge, MA, pp. 123-142.

McQuoid, L.M., Galef Jr., B.G., 1993. Social stimuli influencing feeding behaviour of Burmese fowl: a video analysis. Anim. Behav. 46, 13-22.

Patterson-Kane, E., Nicol, C.J., Foster, T.M., Temple, W., 1997. Limited perception of video images by domestic hens. Anim. Behav. 53, 951-963.

Pepperberg, I.M., 1987. Acquisition of the same/different concept by an African Grey parrot (Psittacus erithacus): learning with respect to categories of color shape and material. Anim. Learn. Behav. 15, 423-432.

Premack, D., Premack, A.J., 1983. The Mind of an Ape. W.W. Norton \& Co., New York.

Rensink, R.A., O’ Regan, J.K., Clark, J.J., 1997. To see or not to see: the need for attention to perceive changes in scenes. Psychol. Sci. 8, 368-373. 
Santiago, H.C., Wright, A.A., 1984. Pigeon memory: same/ different concept learning, serial probe recognition acquisition, and probe delay effects on the serial-position function. J. Exp. Psychol. Anim. Behav. Process. 10, 498-512.

Shimizu, T., 1998. Conspecific recognition in pigeons (Columba livia) using dynamic video images. Behaviour 135, 43-53.

Simons, D.J., 2000. Current approaches to change blindness. Vis. Cogn. 7, 1-15.

Simons, D.J., Levin, D.T., 1998. Failure to detect changes to people during a real-world interaction. Psychon. Bull. Rev. 5, 644 649.

Thompson, R.K.R., Oden, D.L., Boysen, S.T., 1997. Languagenaive chimpanzees (Pan troglodytes) judge relations between relations in a conceptual matching-to-sample task. J. Exp. Psychol. Anim. Behav. Process. 23, 31-43.

Tulving, E., 1983. Elements of Episodic Memory. Oxford University Press, New York.
White, K.G., 2002. Psychophysics of remembering: the discrimination hypothesis. Curr. Dir. Psychol. Sci. 11, 141-145.

Wright, A.A., 1997. Concept learning and learning strategies. Psychol. Sci. 8, 119-123.

Wright, A.A., 2001. Learning strategies in matching-to-sample. In: Cook, R.G. (Ed.), Avian Visual Cognition [On-line]. Available: http://www.pigeon.psy.tufts.edu/avc/wright/.

Young, M.E., Wasserman, E.A., 2001. Stimulus control in complex arrays. In: Cook, R.G. (Ed.), Avian Visual Cognition [On-line]. Available: http://www.pigeon.psy.tufts.edu/avc/young/.

Young, M.E., Wasserman, E.A., Dalrymple, R.M., 1997. Memorybased same-different conceptualization by pigeons. Psychon. Bull. Rev. 4, 552-558.

Young, M.E., Wasserman, E.A., Hilfers, M.A., Dalrymple, R., 1999. The pigeon's variability discrimination with lists of successively presented visual stimuli. J. Exp. Psychol. Anim. Behav. Process. 25, 475-490. 